\title{
Disputas en el orden simbólico: orquestas infantiles y juveniles en Argentina*
}

\section{Karen Avenburg}

\author{
Universidad Nacional de Avellaneda, Avellaneda, Argentina. \\ Correo electrónico: kavenburg@undav.edu.ar \\ Doctora en Ciencias Antropológicas por la Universidad de Buenos Aires; docente e investigadora de la \\ Universidad Nacional de Avellaneda
}

Recibido:

17 de enero de 2018

Aceptado:

27 de junio de 2018

\section{Resumen}

Existen a lo largo del mundo numerosas orquestas infantiles y/o juveniles. Si bien muchas de ellas coinciden en desarrollar la enseñanza musical por medio de la formación orquestal enfocándose en poblaciones en situación de vulnerabilidad, se trata de un campo que está lejos de ser homogéneo. Argentina presenta un escenario particular por la cantidad, dinamismo y diversidad de iniciativas. En este artículo me propongo describir y reflexionar sobre dos programas/proyectos de orquestas infantiles y juveniles de gestión pública, centrándome en las perspectivas que sustentan sus coordinadores: el Programa Social Andrés Chazarreta (Ministerio de Cultura de la Nación) y el Proyecto Orquestas Infantiles y Juveniles (Ministerio de Educación de la Ciudad de Buenos Aires). Sugiero que, si bien se trata de dos proyectos/programas que implican diseños y líneas de acción distintos, ambos apuntan - desde sus diferentes paradigmas - a desestabilizar un orden simbólico que favorece relaciones de desigualdad y exclusión.

\section{Constesting the symbolic order: child and youth orchestras in Argentina}

\author{
Abstract \\ A large number of child and youth orchestras represent initiatives whereby \\ music is taught through orchestral training, focusing on populations in situa- \\ tions of vulnerability. Projects with similar characteristics exist throughout the \\ world, yet this field is far from homogeneous. The specificity of the Argentinean

\section{Palabras clave}

Orquestas infantiles y juveniles; Políticas culturales; Desarrollo simbólico; Música; Inclusión/ exclusión social

Key words

Child and youth orchestras; Cultural policies; Symbolic order; Music; Social inclusion/exclusion 
scenario is characterized by the large number of projects being implemented, their diversity and dynamics. The present article describes and reflects on two state-managed programs / projects for child and youth orchestras, focusing specifically on their coordinators' perspectives: The Social Program "Andres Chazarreta" (National Ministry of Culture) and the Child and Youth Orchestras Project (Ministry of Education of the City of Buenos Aires). I suggest that although these initiatives involve different designs and lines of action, they both contribute, from their different paradigms, to destabilize a symbolic order that fosters relations of inequality and exclusion.

\section{Disputando a ordem simbólica: orquestras infantis e juvenis na Argentina}

\section{Resumo}

Palavras-chave

Orquestras infantis e juvenis; Políticas culturais; Ordem simbólica: Música Inclusão/exclusão social
1. Existen investigaciones sobre este tipo de proyectos a nivel internacional; sobresalen por el alcance y la cantidad aquellas que observan el Sistema Nacional de Orquestas y Coros Juveniles e Infantiles de Venezuela - fundado por José Antonio Abreu en los años setenta- (Borchet, 2012; Majno, 2012; Tunstall, 2012; Uy, 2012; Baker, 2014). (continúa en página 111)
Uma grande quantidade de orquestras infantis e juvenis representam iniciativas que desenvolvem o ensino musical através da formação orquestral com foco nas populações em situação de vulnerabilidade. Embora existam projetos com caraterísticas similares ao longo do mundo, trata-se de um campo que está longe de ser homogêneo. Argentina apresenta um cenário particular pela grande quantidade, dinamismo e diversidade de projetos. Neste artigo eu proponho a descrever e refletir sobre dois programas/projetos de orquestras infantis e juvenis de gestão pública, focando especificamente nas perspectivas que sustentam suas formulações: o Programa Social Andrés Chazarreta (Ministério de Cultura da Nação - Argentina) e o Proyecto Orquestas Infantiles y Juveniles (Ministério de Educação da Cidade de Buenos Aires). Eu sugiro que, embora sejam projetos/programas que envolvam concepções bem diferentes no tocante à política cultural, ambos contribuem, desde seus próprios paradigmas, a desestabilizar uma ordem simbólica que favorece relações de desigualdade e exclusão.

\section{Introducción}

Los proyectos o programas de orquestas infantiles y juveniles son, en muchos casos, conjuntos de acciones diseñadas y ejecutadas por agentes de diversos sectores - públicos, privados o del tercer sector - que desarrollan la enseñanza musical mediante la formación orquestal, enfocándose en poblaciones en situación de vulnerabilidad. Si bien pueden desenvolverse de diversas maneras y desde concepciones muy diferentes, estos proyectos suelen coincidir en el empleo de la práctica orquestal como parte integral del proceso de enseñanza, sumado a lo que en la agenda pública se ha denominado inclusión social término que se encuentra en discusión (ver, por ejemplo, Pérez Rubio, 2006) -; a veces se menciona la integración y/o transformación social (ver Avenburg, Cibea y Talellis, 2017). Existen muchas iniciativas con estas características en el mundo. Se puede mencionar, por ejemplo, a Brasil, Chile, Colombia, Costa Rica, Ecuador, Escocia, México, Paraguay, Sudáfrica, Uruguay, Venezuela, ${ }^{1}$ y también a la Argentina (sobre los proyectos y programas locales, ver Wald, 
2009, 2011 y 2017; Villalba, 2010; Fernández Calvo, s/f; Muiños, 2010; Martínez, 2016; Vázquez, 2016).

Como telón de fondo de estos proyectos podemos pensar a las coyunturas sociales y económicas de finales del siglo XX, producto de la instrumentalización de un esquema neoliberal que tuvo como consecuencia un aumento de la exclusión social. En los años noventa, en gran parte de América Latina se implantaron políticas de desregulación económica, ajuste fiscal, privatizaciones, desindustrialización, reducción de políticas sociales y concentración de bienes simbólicos y materiales, que implicaron la expulsión del mercado de grandes cantidades de personas, la degradación de las condiciones de vida de amplios sectores de la población, el aumento de la desigualdad social, y el surgimiento de nuevas formas de marginalidad y exclusión (Villarreal, 1996; Pérez Rubio, 2006; Svampa, 2010; Kliksberg, 2014). El aumento de la exclusión social producida - también en Europa - por las medidas neoliberales de los años ochenta y noventa se considera relevante para entender el devenir de las políticas culturales que en las últimas décadas se volcaron hacia problemáticas sociales, económicas o educativas, entre otras (Belfiore, 2002; Barbieri, Partal y Merino, 2011) $)^{2}$.

Efectivamente, en el ámbito local, las orquestas, proyectos y programas que continúan vigentes se remontan a fines de la década del noventa (Avenburg, Cibea y Talellis, 2017): la Orquesta Infantil y Juvenil de la SENNAF, creada en 1996 (dependiente de la Secretaría Nacional de Niñez, Adolescencia y Familia del Ministerio de Desarrollo Social de la Nación, y del Programa Social de Orquestas y Bandas Infantiles y Juveniles del Ministerio de Cultura de la Nación); la Orquesta de Lugano, creada en 1998 (Proyecto Orquestas Infantiles y Juveniles del Ministerio de Educación de la Ciudad Autónoma de Buenos Aires); y la Orquesta-Escuela de Chascomús, creada en 1998 (Núcleo inicial de la Fundación Sistema de Orquestas Infantiles y Juveniles de Argentina).

Si bien se desarrollan iniciativas con algunas características similares en todo el mundo desde hace ya varias décadas, se advierten escenarios particulares vinculados a características sociales, territoriales y políticas locales. Una de las especificidades del panorama argentino es la existencia de múltiples proyectos y programas públicos (nacionales, provinciales, municipales), privados y del tercer sector que, a su vez, despliegan diversas articulaciones entre sí. En el relevamiento realizado con el Grupo de Investigación sobre Música e Inclusión $^{3}$ pudimos observar esto en detalle en el Gran Buenos Aires, donde, a finales del año 2015 existían 116 orquestas: 36 ubicadas en la Ciudad Autónoma de Buenos Aires y 80 distribuidas en los partidos del Gran Buenos Aires (Avenburg et al., 2015). La mayoría de ellas están insertas en proyectos o programas pertenecientes a diferentes instituciones públicas de nivel nacional, provincial o local bajo distintas dependencias institucionales, pero también hallamos varios proyectos pertenecientes al tercer sector y unos pocos del ámbito privado. Este panorama se enriquece en virtud de las diversas y muy dinámicas articulaciones posibles, pues las combinaciones van desde la gestión única hasta la gestión articulada entre diferentes sectores (público, privado y tercer sector) y, dentro del sector público, entre distintos niveles administrativos (nacional, provincial y local) y entre diversas áreas de políticas públicas (Cultura, Educación o Desarrollo Social, etc.) (Avenburg, Cibea y Talellis, 2017).

Cada uno de estos programas o proyectos desarrolla sus propias concepciones acerca de la música, la cultura, la inclusión, la integración y/o transformación social, la política cultural y las poblaciones involucradas, que impactarán en
2. Pueden hallarse también diversas iniciativas que desarrollan otras actividades artísticas como prácticas de inclusión, integración o transformación social, tales como teatro (Sánchez, 2014), circo (Infantino y Raggio, 2007; Infantino, 2011), danza (Lloret, 2009; Greco e luso, 2012) y artes plásticas (Dillon, 2008; Dillon y Deluca, 2012), entre otras.

3. Es un equipo interdisciplinario de investigación radicado en la Universidad Nacional de Avellaneda, compuesto por investigadoras formadas y en formación de las áreas de Antropología, Ciencias Políticas, Gestión Cultural, Etnomusicología, Pedagogía Musical y Periodismo. (continúa en página 111) 
4. Desde ya, los diferentes integrantes de un mismo proyecto pueden también sustentar posiciones diversas -en general, esto ocurre dentro de ciertas ideas compartidas-. (continúa en página 111)

5. En efecto, Susan Wright (2007) nos insta a entender la cultura como procesos activos de construcción y disputa por los significados.

6. El corpus en el que se sustenta la investigación de la que es parte este trabajo excede las fuentes aquí empleadas e incluye, desde un enfoque etnográfico, (continúa en página 111)

7. En esta mesa, referentes de diversos proyectos y programas de orquestas discutieron y pusieron en diálogo los procesos de creación, los desarrollos y las dinámicas cotidianas que implica gestionar este tipo de iniciativas. (continúa en página 111) el diseño y puesta en práctica de las orquestas. ${ }^{4}$ A la vez, este diseño y puesta en práctica se modificarán en función de las expectativas y experiencias de los destinatarios, así como de su articulación con lineamientos de políticas públicas coyunturales - especialmente en el caso de proyectos estatales-. Todo esto redundará en la generación de variaciones entre diseño y acción de un proyecto o programa y, dentro de cada uno de ellos, entre diferentes orquestas. En consecuencia, pese a que a veces se habla de las orquestas infantiles y juveniles como un todo homogéneo, se trata de iniciativas dinámicas, heterogéneas, complejas y que se constituyen - al igual que todo fenómeno cultural - en campo de disputas. ${ }^{5} \mathrm{Si}$ bien entonces podemos señalar varios puntos en común, existen diferencias que hacen que cada uno de ellos despliegue sus propias particularidades.

En este artículo me propongo describir un programa y un proyecto de orquestas infantiles y juveniles de gestión pública que se desarrollan en Argentina, centrándome específicamente en las perspectivas que sustentan integrantes de sus equipos de coordinación - y que se traducen en sus diseños y puestas en acción-: el Programa Social Andrés Chazarreta (Ministerio de Cultura de la Nación) y el Proyecto Orquestas Infantiles y Juveniles (Ministerio de Educación de la Ciudad de Buenos Aires). La elección de estos dos casos responde a que ambos son de gestión pública, pero en dos niveles diferentes uno nacional y otro local $-\mathrm{y}$ pertenecientes a dos áreas de gestión distintas -Cultura y Educación - . Para ello me basaré tanto en entrevistas realizadas con diferentes integrantes de sus equipos de coordinación, como en observaciones y charlas informales en diversas situaciones - conciertos, manifestaciones, encuentros de discusión.$-{ }^{6}$ Las citas textuales que se despliegan en los próximos apartados provienen de entrevistas en profundidad y semiestructuradas, comunicaciones personales (algunas de ellas revisando fragmentos de este escrito), y una mesa redonda organizada por el Grupo de Investigación sobre Música e Inclusión, denominada "Los proyectos y programas de Orquestas Infantiles y Juveniles". Esta última se desarrolló en octubre del año 2015 en el marco del Encuentro por el Día Nacional de la Gestión Cultural en la Universidad Nacional de Avellaneda. ${ }^{7}$

\section{Algunas especificaciones conceptuales: políticas públicas, políticas culturales, inclusión/exclusión social}

El concepto de inclusión/exclusión social fue desarrollado por la sociología francesa en los años sesenta; luego se expandió progresivamente a otras regiones, y contribuyó al análisis de las desventajas sociales en el marco de los cambios socioeconómicos asociados a la declinación de los Estados de Bienestar (Castel, 1997; Fitoussi y Rosanvallon, 1997; Belfiore, 2002; Pérez Rubio, 2006; Svampa, 2010). Esto no significa que los fenómenos vinculados con la desigualdad, desafiliación o exclusión fueran una novedad sino, como sostiene Robert Castel (1997), que se trata de la "metamorfosis" de una misma problematización, la de "la cuestión social". Si bien hay amplias y ricas discusiones conceptuales en torno a estos términos y otros emparentados (ver por ejemplo Castel, 1997; Autes, 2004; Karsz, 2004; Pérez Rubio, 2006), se destaca que el desarrollo de estas nociones puso en foco que la exclusión social no refiere exclusivamente al plano material, sino que posee también dimensiones sociales y culturales (Villarreal, 1996; Belfiore, 2002). Se observa además que no es un problema individual de los grupos situados en los márgenes, sino producto de la configuración del Estado y de la dinámica de relaciones sociales (Castel, 1997; Pérez Rubio, 2006). Lo antedicho conduce a una concepción de 
la problemática de la inclusión/exclusión social tanto en términos de acceso a recursos materiales, al mercado de trabajo, etc., como de inscripción en las redes sociales y de participación en la producción simbólica. Se consideran sus dimensiones étnicas, socioeconómicas, migratorias y culturales (Villarreal, 1996; Belfiore, 2002), pues se detecta que hay grupos privados de derechos laborales, educativos y culturales, entre otros.

Esta perspectiva de la problemática de la inclusión/exclusión social, que involucra, entre otras cosas, las relaciones sociales y la producción simbólica de la sociedad como conjunto, posibilita pensar en políticas públicas en el campo cultural. De acuerdo con Néstor García Canclini (1987: 26), las políticas culturales se entienden como "el conjunto de intervenciones realizadas por el Estado, las instituciones civiles y los grupos comunitarios organizados a fin de orientar el desarrollo simbólico, satisfacer las necesidades culturales de la población y obtener consenso para un tipo de orden o de transformación social". Si bien los agentes que gestionan políticas culturales pueden ser diversos, el lugar del Estado es central, pues no hay régimen político sin estrategias que orienten el desarrollo simbólico de la sociedad (García Canclini, 1987; Olmos, 2008). Se tornan relevantes entonces las políticas públicas, entendidas como la "toma de posición y curso de acción adoptados por un actor que habla en nombre y representación del estado, frente a una cuestión socialmente problematizada" (Oszlak, 2009: 5, nota al pie).

Es relevante, por ende, atender a las perspectivas de los coordinadores de proyectos o programas públicos. Desde ya estas no son las únicas que delinean políticas y cursos de acción; pero como observa Liliana Raggio (2013) retomando a Oszlak y O'Donnell (1981), si las políticas públicas plasman respuestas a necesidades que diversos grupos, en contextos específicos, logran transformar en demandas, hay por debajo disputas por incluir significados y necesidades, y por transformarlos en derechos y normativas. En este contexto, las definiciones de los funcionarios y las intervenciones del Estado tienen un peso privilegiado - aunque no único ni indiscutido- para promover perspectivas y prácticas en la sociedad. En coincidencia con Rubens Bayardo (2010), las políticas culturales ofrecen representaciones del mundo que sesgan tanto las maneras en que los diferentes grupos humanos pueden verse a sí mismos y a los demás, como las posibilidades de tomar decisiones y actuar.

Se desprende de lo antedicho la pregunta en torno al universo simbólico que se busca construir, reforzar o cuestionar al diseñar proyectos o programas como parte de una política cultural pública. La perspectiva de quienes se ocupan de crear, diseñar y poner en acción un proyecto, programa o plan específico puede dar lugar a representaciones y desarrollos diferentes. En el caso de las orquestas infantiles y juveniles, se puede observar la manera en que unas políticas que apuntan a la formación musical por medio de la práctica orquestal con foco en poblaciones en situación de vulnerabilidad tienen la doble tarea de desplegar acciones concretas de formación artística, al tiempo que buscan actuar en el campo social y cultural de maneras diversas para atender a situaciones vinculadas a los procesos de exclusión social. Si las examinamos como políticas culturales, ${ }^{8}$ ¿qué tipo de desarrollo simbólico buscan estas iniciativas?; ¿qué sistemas de ideas acompañan y con qué sistemas de ideas confrontan? A partir de estos interrogantes, me propongo aquí reflexionar acerca de un proyecto y un programa ${ }^{9}$ de orquestas atendiendo a las perspectivas de quienes los coordinan; describiré primero cada a uno de ellos para luego reflexionar acerca del modo en que, desde ángulos diferentes,
8. María Villalba (2010) realiza un interesante análisis de dos programas de orquestas como políticas públicas culturales, educativas y sociales, centrándose especialmente en estas últimas.

9. Si bien, como explican los mismos actores, un programa abarca un conjunto de proyectos bajo su órbita, mientras que un proyecto es más acotado, (continúa en página 111) 
buscan disputar un orden simbólico que favorece relaciones de desigualdad y exclusión.

\section{El Programa Social Andrés Chazarreta}

10. Andrés Chazarreta (1876-1960) fue un músico, docente e investigador santiagueño dedicado a la composición, recopilación y difusión de música folklórica argentina.

11. De acuerdo con integrantes del programa, el despido de Eduardo

Tacconi a fines de 2017 pone en

duda la continuidad de aquel.

(continúa en página 112)Esto

12. En el rastreo realizado con

el Grupo de Investigación sobre Música e Inclusión (Avenburg et al., 2015), reconstruimos los objetivos,

la población destinataria y el repertorio de diferentes proyectos y programas. (continúa en página

13. En Avenburg, Cibea y Talellis (2017) nos referimos a las distintas modalidades en las que se coordinan diferentes agentes en términos de gestión articulada, entre las que incluimos diversas variantes de combinaciones entre actores. (continúa en página 112)

14. A fin de mantener la confidencialidad, tanto de las entrevistas como de las intervenciones en la mesa redonda en un contexto complejo y atravesado por fuertes tensiones, no se consignan los nombres de los interlocutores, sino los roles que cumplían en el momento de su realización (cabe aclarar que algunos integrantes del equipo de coordinación del programa dejaron o fueron alejados de dichos roles desde finales de 2017).
El Programa Social Andrés Chazarreta ${ }^{10}$ depende de la Dirección Nacional de Diversidad y Cultura Comunitaria - hasta hace unos años formaba parte de la Dirección Nacional de Artes - del Ministerio de Cultura de la Nación. La coordinación general estuvo a cargo de Eduardo Tacconi desde sus inicios, en el año 2006, hasta finales de 2017. ${ }^{11}$ Se desarrolla a lo largo del territorio nacional y tiene como población destinataria a "Niños, niñas, adolescentes y jóvenes de Argentina. Se hace hincapié en los sectores históricamente marginados del acceso a políticas públicas de prácticas musicales" (Programa Andrés Chazarreta, citado en Avenburg et al., 2015). ${ }^{12}$

Funciona en una modalidad de gestión articulada, ${ }^{13}$ que puede ir de la articulación del sector público de nivel nacional con grupos del tercer sector, hasta la gestión pública de otros niveles de administración (provincial, municipal) y de diversas áreas de políticas públicas. El programa, por lo general, suministra los instrumentos, proporciona diversas herramientas pedagógicas y brinda capacitaciones, y la contraparte se ocupa de los sueldos y del espacio necesario para desarrollar la actividad - aunque dependiendo de las necesidades de cada caso, esta modalidad puede variar - . Cuenta con 31 orquestas en la actualidad a lo largo del territorio argentino. Se desarrolla entonces por medio de convenios con otros sectores, áreas y niveles del Estado:

estos convenios nos aseguran, por un lado, la continuidad y un contacto directo en el territorio. Nosotros nos encargamos de proveer instrumentos periódicamente, primero una entrega general como para un inicio, que siempre estamos como tratando de actualizarlo, de mejorar los materiales, y el municipio o la contraparte, digamos, se encarga de proveer un lugar para que funcione. En general son escuelas también, o centros de integración comunitaria, en fin, diversos, como que cada orquesta es también dependiendo del lugar donde funciona... (integrante del Equipo de Coordinación, Mesa Redonda - 2015). ${ }^{14}$

La diversidad y complejidad del territorio que abarca este programa obliga a tener cierta flexibilidad en cuanto a la modalidad de articulación; como explicaba el mismo integrante del Equipo de Coordinación en la mesa redonda (2015):

el programa no está unificado desde quién contrata a los trabajadores, sino que tiene que ver más con la idea esta de la enseñanza colectiva como eje, enseñanza colectiva de música popular, (...) porque después cada caso es muy particular, y también se hace dependiendo de las necesidades, de las posibilidades, bueno, todos los contratiempos.

\section{Cuestionamientos a la formación eurocéntrica}

En tanto discute la colonialidad de las modalidades de enseñanza de diversos espacios de formación musical, este programa se planteó en torno a distintas consideraciones que incluyen dimensiones pedagógicas, sociales y culturales, y se articulan en una mirada crítica hacia la formación y la práctica musical centradas en tradiciones musicales europeas. Por un lado, se enfatiza la capacidad pedagógica de la enseñanza colectiva: “¿Cuál es nuestra principal crítica a los institutos de enseñanza superior? La enseñanza individual. Muchos 
estamos convencidos de que la enseñanza colectiva de los instrumentos y de la música es superadora, es mucho más ágil" (Entrevista a integrante del Equipo de Coordinación - 2015). Por otro lado, se destaca el carácter irrestricto del ingreso, como afirma el mismo interlocutor: "Nosotros estamos convencidos de que no debe haber selección de aptitudes. No hay que elegir el/la niño/a que tiene oído o el que no tiene, no es cierto, en un 98 por ciento de los casos estás cortándole la posibilidad de expresión a un montón de gente que no tiene problemas".

También en el plano pedagógico se pone en primer plano la percepción sonora:

Hay una propuesta, sí, que es esta del aprendizaje colectivo desde lo oral como punto de partida, que los chicos tengan un contacto directo con la música, y no quizá de movida ya con un intermediario, que es el papel digamos, más allá de que para mí es una herramienta que está buenísima, y que permite ampliar... (integrante del Equipo de Coordinación, Mesa Redonda - 2015).

Esto se debe a que, como dice otro de sus integrantes, "como partimos de un programa de música popular, y las músicas populares nacen de tocar, de jugar, de encontrarse, de bailar; tratamos de que se mantenga ese origen, ese origen en la forma de enseñarlo" (integrante del Equipo de Coordinación y director de una Orquesta del Programa, Mesa Redonda - 2015). Esto se basa en un enfoque pedagógico que toma a la percepción sonora como punto central y a la notación como herramienta. Como explica un integrante del Equipo de Coordinación (Comunicación personal, 2017), al trabajar desde géneros musicales vivos y culturalmente cercanos, sus usos y desarrollos se piensan a partir de versiones; esto se facilita desde la oralidad, en contraposición con expresiones musicales "de autor", que se ejecutan siguiendo la partitura ${ }^{15}$ y tal como fueron armadas.

De lo antedicho se vislumbran otras dos particularidades que distinguen especialmente a este programa y que articulan la mencionada mirada crítica al eurocentrismo: el repertorio y los instrumentos empleados. El primero comprende "las especies vivas de la música argentina y latinoamericana" (página Web del programa: https://chazarreta.cultura.gob.ar), tales como candombe, chacarera, cumbia, gato, guarania, huayno, merengue, vals, vidala, xote, zamba, entre otros. Los segundos siguen esa misma perspectiva e incluyen instrumentos de cuerda armónicos (tales como guitarras, charangos, guitarrones y, en ocasiones, cuatro venezolano, mandolina y bandola), un set de percusión latinoamericana (conga y bombo legüero, entre otros), cuerdas de arco (como violines y contrabajos) y aerófonos de caña y madera (quena, sicus, moceños) además de flautas traversas y acordeones. Como explica uno de sus integrantes,

El Chazarreta surge como una propuesta de enseñar música popular, particularmente música argentina, música latinoamericana, desde el repertorio y desde la formación, desde los instrumentos, que esa quizás es la segunda diferencia más grande con programas que tienen por ahí una raíz más en lo académico (...), como de origen europeo digamos (integrante del Equipo de Coordinación, Mesa Redonda - 2015).

Como se puede ver en los instrumentos mencionados, se incluyen aquellos procedentes de la historia musical occidental apropiados desde la experiencia musical local:

Los instrumentos (...) algunos son de origen europeo, otros son de origen americano, pero sí todos los instrumentos que se eligen son instrumentos que históricamente,
15. Si bien se parte de la práctica oral como propuesta pedagógica, hay flexibilidad para introducir la notación musical cuando se hace necesaria para los estudiantes o los docentes. 
16. El resaltado es de quien habla (en comunicación previa a esta publicación). en los últimos siglos, tienen un desarrollo claro y probado digamos en distintas músicas de nuestro territorio. Desde ahí viene el enfoque (integrante del Equipo de Coordinación y director de una Orquesta del Programa, Mesa Redonda - 2015).

El repertorio sigue esta misma línea e intenta combinar las particularidades poblacionales y regionales con su pertenencia a un territorio más amplio y diverso: "lo regional muchas veces sucede espontáneamente, o sea, una orquesta que se arma en el litoral tiene su dosis de chamamé, de rasguido doble, de músicas de la zona. Pero promovemos nosotros que esté eso y que haya una visión más latinoamericanista" (integrante del Equipo de Coordinación y director de una Orquesta del Programa, Mesa Redonda - 2015). Esta perspectiva latinoamericanista apunta también a que orquestas que se desarrollan en comunidades con personas de diferentes procedencias puedan ver reflejada y respetada esa diversidad. Es el caso de las orquestas situadas en barrios que abarcan poblaciones heterogéneas; el mismo interlocutor daba como ejemplo algunas que incluyen chicos procedentes de Bolivia, Perú y Paraguay, y muchos migrantes internos. Según explicaba,

está bueno para nosotros trabajar con eso, porque ponemos el eje también en la revalorización digamos de la identidad cultural, por lo que debe significar para ellos y para sus familias haberse ido de su tierra, de su cultura, y de todo eso, a vivir en una villa en la Capital. Y para nosotros revalorizar esa identidad desde la música, nos parece, para los chicos, fundacional, un eje importante (integrante del Equipo de Coordinación y director de una orquesta del Programa, Mesa Redonda - 2015).

\section{Una mirada transversal atenta a la diversidad}

En el diseño de este programa como política cultural pública se traduce la perspectiva del equipo que lo generó y lo lleva adelante. Se considera fundamental desarrollar y difundir expresiones y prácticas que no se despliegan en los medios hegemónicos: “¿El Estado qué tiene que hacer? Poner lo que no hay; más si es genuino. (...) Entonces para nosotros desde el Estado teníamos que poner lo que no había, por eso pusimos el Chazarreta". ¿Por qué esta música y no otras? "Porque el eje tiene que ser para quién lo hacemos, ${ }_{16}^{16}$ y si nosotros queremos abordar como sujetos a los niños de todo este país, que es tan diverso, tenemos que tratar de encontrar cosas que transversalmente interesen a todos esos grupos, salirnos del esquema porteño" (Entrevista a integrante del Equipo de Coordinación - 2015).

Los objetivos del programa sintetizan buena parte de lo antedicho: ¿qué es lo que se busca? "Proporcionar a los niños, niñas, adolescentes y jóvenes de zonas vulnerables el acceso a la práctica musical colectiva sin selección de aptitudes, en este caso, en base a la música argentina y latinoamericana" (Programa Andrés Chazarreta, citado en Avenburg et al., 2015). Según explica el integrante recién citado,

Para mí, el objetivo básico de estos programas desde el Estado tiene que ser ampliar la oferta pública de aprendizaje y práctica musical para nuestra infancia y juventud (...). Entonces ampliar la oferta considerando la música vinculada con el canto y la danza, elementos insustituibles del desarrollo humano. (...) Bueno, hay mucho más para hablar, pero cualquier persona que en la infancia esté desarrollando una tarea de estas claramente en general mejora su relación con todo el entorno, familiar, social y del aprendizaje (Entrevista a integrante del Equipo de Coordinación - 2015). 
Al discutir la noción de inclusión y las perspectivas paternalistas, explica que se trata de una búsqueda de justicia social respetuosa de la diversidad de las comunidades destinatarias:

¿qué significa inclusión social? ¿Es incluir al otro a dónde, en qué mundo? Entonces para mí justicia social... (...) Las palabras nunca expresan todo, pero el concepto ya habría que ponerlo en cuestión, porque es justamente lo que da lugar a veces a la tergiversación, "yo le llevo la cultura a la gente", cuando en realidad yo tengo que respetar su pertenencia cultural, y a lo sumo facilitar herramientas, y si tengo más conocimientos específicos de algo ser lo suficientemente elástico para ver... Porque los chicos tienen padres, y madres, y hermanos, y comunidad, es una irrespetuosidad ir a plantearles "yo tengo la sabiduría".

Se advierte aquí una de las discusiones que giran en torno al concepto de inclusión social. Dentro de sus muchas aristas y acepciones, es significativa aquella que implica el cuestionamiento de la estructura en la que se desarrollan las prácticas que se esperan inclusivas. Al objetar la estructura, se pone en cuestión la misma idea de inclusión (pues se plantea la siguiente pregunta: ¿por qué querer incluir en algo que está mal en sus bases?). ${ }^{17}$

En suma, desde diferentes aspectos, el Programa Chazarreta apunta a la enseñanza colectiva en un desarrollo transversal, atento a la diversidad y a las particularidades de la población destinataria, y desde un cuestionamiento del eurocentrismo en las formas de enseñanza, en los contenidos transmitidos y en las perspectivas sobre la música y la cultura. La misma estructura organizativa del programa refleja esta perspectiva, pues ha funcionado desde sus inicios con un núcleo conformado por integrantes de todo el país, en cuyo marco las acciones se deciden entre todos.

\section{El Proyecto Orquestas Infantiles y Juveniles}

Este proyecto - que tiene las dimensiones de un programa - fue uno de los primeros en crearse que aún continúan vigentes; se inició en el año $1998^{18}$ dentro del Programa ZAP (Zonas de Acción Prioritaria), de la entonces Secretaría de Educación del Gobierno de la Ciudad de Buenos Aires. Actualmente depende de la Gerencia Operativa de Recorridos Educativos (Dirección General Escuela Abierta, Subsecretaría de Equidad Educativa) del Ministerio de Educación de la Ciudad de Buenos Aires, y cuenta con 16 orquestas. Desde sus inicios, la coordinación general está a cargo de Claudio Espector, cofundador del proyecto. Este tiene, entre sus muchos objetivos: "-Incorporar nuevos sectores sociales al proceso educativo musical. -Promover y estimular la cultura musical en el seno de estas comunidades. -Sumar las orquestas infantiles a los programas desarrollados en áreas históricamente postergadas. (...) -Promover y desarrollar la práctica orquestal como actividad conjunta, cooperativa, comenzando a tocar un instrumento junto a otros, aprendiendo junto con otros" (Proyecto Orquestas Infantiles y Juveniles, citado en Avenburg et al., 2015).

El proyecto se articula en torno a una serie de ideas que se podrían sintetizar en la búsqueda de posibilitar el acceso y la apropiación por parte de niñas, niños y jóvenes de un bien cultural socialmente valorado, como es la música, teniendo como telón de fondo un contexto de desigualdad.
17. Por el otro lado se puede pensar que la inclusión cambia esas mismas bases (al cambiar su carácter excluyente). El campo de discusión es amplio, solo pretendo aquí reflejar algunas de sus muchas vetas.
18. Comenzó con la Orquesta de Lugano, que progresivamente se fue dividiendo en tres (inicial, intermedia y avanzada). Unos años después (2002) se creó la Orquesta de Retiro, también dividida luego en inicial y avanzada. Posteriormente se fueron abriendo más orquestas hasta llegar a las dimensiones que mantiene en la actualidad. En el Informe Final de la Investigación sobre las Orquestas Infantiles de Villa Lugano de Antelo y Zanelli (2004) se puede encontrar una descripción reflexiva de los inicios del proyecto, junto con sugerentes discusiones de algunos conceptos y problemáticas que surgen en torno a estas iniciativas. 
El repertorio, por ejemplo, abarca "Principalmente música académica y popular. Se incluye cualquier género musical siempre que los arreglos posibiliten que todos los chicos puedan tocar" (Proyecto Orquestas Infantiles y Juveniles, citado en Avenburg et al., 2015). Entre ellos se cuentan expresiones como carnavalito, cumbia, rock, música de películas, música europea del Clasicismo y el Romanticismo, etc. A diferencia del programa antes presentado, la formación instrumental es la de la orquesta sinfónica - con instrumentos de procedencia europea-: se incluyen instrumentos de cuerda (violines, violas, cellos, contrabajos), instrumentos de viento de madera (flauta travesera, clarinete, oboe), instrumentos de viento de metal (trompeta, corno, trombón, fliscorno) y percusión (xilófono, glockenspiel, timbales, tambor, bombo, platillos y accesorios). En este caso, de forma paralela al aprendizaje del instrumento, los chicos tienen también clases de lenguaje musical, pues en este proyecto se trabaja principalmente y desde el inicio con base en la lectoescritura musical.

Según explica un integrante del Equipo de Coordinación (Entrevista - 2014), con este proyecto se busca

distribuir un cierto bien social y cultural, que es la música, que es la posibilidad de tocar un instrumento, de ser parte de una orquesta, y eso se plantea en (...) un contexto de desigualdad, por decirlo de alguna manera, donde evidentemente hay sectores de la población que por su situación socioeconómica le son sesgados, y tradicionalmente sesgados. Y se parte de un convencimiento que tiene que ver con que estos bienes o esta enseñanza es una enseñanza que tiene que ser de calidad y que eso no puede ser en detrimento de la cuestión esta más social, por decirlo de alguna manera, de las orquestas.

Esta intencionalidad de apuntar a sectores históricamente postergados se refleja en la población destinataria del proyecto: “Niños, niñas y jóvenes que asisten a escuelas ubicadas en zonas de vulnerabilidad social de diversas jurisdicciones de la Ciudad de Buenos Aires" (Proyecto Orquestas Infantiles y Juveniles, citado en Avenburg et al., 2015). Como el Programa Chazarreta, también aquí el carácter del ingreso es irrestricto. Se advierte nuevamente la búsqueda de facilitar el acercamiento a una práctica cultural a sectores postergados de la población; como explica una exintegrante (actualmente jubilada), se considera que el desarrollo de esta práctica musical tiene efectos positivos en los participantes - de ahí la importancia de facilitar su acceso-:

En realidad, una de las cosas más interesantes de este proyecto es que no importa que los chicos estén dotados para la música, ni que tengan buen oído, ni que sean sensibles. ¿Quieren hacer música? Vos vení. El chico aprende a tocar dos notas y ya va al ensayo de orquesta y toca esas dos notas. Y es interesantísimo desde el punto de vista pedagógico pensar que ese chico aprende desde ese primer momento que tiene que estar atento al director, que tiene que escuchar todo lo que pasa a su alrededor, que él va a tener que entrar en un momento determinado y tiene que estar muy atento. Bueno, todo esto es fundamental para estructurar su personalidad (...). Creo que a partir de ahí es un puntapié para mejorar los aprendizajes, para mejorar su relación con la vida, con el otro (Entrevista a exintegrante del Equipo Administrativo - 2014).

En este proyecto también se está pensando en la importancia de su desarrollo como política pública; en palabras de otro interlocutor,

la fundamentación de las orquestas es parte de la universalidad de un discurso, de la universalidad de un derecho, si algo es un derecho es universal; si no, no es un 
derecho. Entonces partimos de eso, hay un bien valorado socialmente que debe ser distribuido. Punto. ¿Cuál es la población? Para todos los chicos y chicas que tengan ganas de participar. Punto y aparte, digamos. Si te quedás en eso entonces ahí decís "bueno, por qué están en los barrios pobres, si es para todos, es para todos". Entonces digamos, una cosa es proclamar que algo es un derecho y otra cosa es después ver como Estado, como política pública, qué hacés al respecto. Entonces se parte también de reconocer un contexto de desigualdad en la distribución de esos bienes, y que está signada no por el azar sino por cuestiones sociales, socioeconómicas, como quieras llamarlas. Entonces en ese contexto es una política pública que de algún modo se plantea reparar de algún modo esa situación; entonces para que eso sea efectivo tenés que llegar a aquellos lugares donde los pibes, por el lugar en el que están -no por una cuestión de territorio geográfico, sino de verdad por una cuestión del lugar donde están-, tienen un acceso mucho más dificultoso a eso. Entonces bueno, funcionamos en aquellos lugares donde los pibes más lo necesitan, por decirlo de alguna manera, y además están en una situación de mayor desigualdad (Entrevista a integrante del Equipo de Coordinación - 2014).

\section{Contra una lógica expulsiva}

El hecho de que se piense en una política pública que ponga el foco en las dificultades generadas por un contexto de desigualdad hace que la búsqueda por distribuir un bien socialmente valorado esté atenta a evitar reproducir modalidades educativas propias de un contexto excluyente. Según explican sus integrantes, se busca romper con el carácter expulsivo de algunas instituciones de la sociedad:

hay una preocupación (...) que es de qué modo hacemos para no reproducir una lógica que evidentemente no... no queremos entrar, esta idea del sistema educativo expulsivo, o de un filtro que se va dando a partir de la situación de algunos pibes. Es decir, si los pibes que por una situación de estar en mayor vulnerabilidad, por decirlo de alguna manera, se empezaban a quedar afuera (Entrevista a integrante del Equipo de Coordinación - 2014).

Otra de las interlocutoras citadas explica una de las maneras en que se busca atender a este problema:

en la orquesta no se echa a nadie, no se va nadie, el que se va se lo busca para que vuelva, los asistentes lo llaman por teléfono, hablan con los padres, a veces se van hasta la casa a buscarlos para ver qué pasó, por qué se va, por qué no viene, qué le está pasando. (...) Y si el chico pasaron cinco meses y vuelve, es bien recibido, no te quedás libre, no perdiste el año, nada, vos vení. ¿Quedaste acá? Bueno, de acá tenemos que seguir avanzando un poco más. ¿Tus compañeros avanzaron mucho más? Bueno, vos despacito vas a lograr... (Entrevista a exintegrante del Equipo Administrativo - 2014).

En su opinión, modalidades como las que se desarrollan en este tipo de proyectos, con su énfasis en lo colectivo y en la combinación de "libertad contenida y de participación", no solamente buscan revertir lo que ocurre en otros contextos institucionales sino que, además, deberían ser consideradas también para esos otros ámbitos educativos:

Este me parece que es el rumbo que en realidad debiera empezar a llevar la educación en general. Yo creo que la educación está absolutamente estancada, que es terrible cómo está encarada, y que ya no es acorde con los tiempos ni con la vida actual, y que hay que empezar a cambiar todo, pero todo profundamente. 
19. Si bien no es un tema a desarrollar aquí, es importante desmitificar las imágenes idealizadas de la música en sí misma. Como observa Small (2010), la tradición musical de Occidente ha tendido a ser jerarquizante y excluyente -a diferencia de otras tradiciones musicales-, íntimamente vinculada a la modalidad capitalista de conocer y de relacionarse con el mundo. En estos proyectos no se está pensando en la música en términos idealizados, sino en determinadas maneras de practicarla y enseñarla.
Y que en orquestas yo encontré (...) la punta de por dónde tiene que ir un poco la cosa. Esta cosa de libertad contenida y de participación, y de conjunto; y que yo puedo estar estudiando muchas horas en mi casa pero lo que yo hice acá lo tengo que demostrar con todos, si no, no sirve. Y bueno, a mí me parece una cosa muy interesante (Entrevista a exintegrante del Equipo Administrativo - 2014).

En efecto, y como ya señalé, no se piensa solamente en la posibilidad de acceso a determinadas prácticas culturales, sino en que dichas prácticas, con los modos de relación específicos que se buscan desplegar, ${ }^{19}$ se piensan en términos positivos. Por ejemplo, el modo de enseñanza también aquí se centra en lo colectivo:

El trabajo está propuesto desde lo grupal, no desde lo individual, se trabaja por grupos en todos los instrumentos, y esto va creando (...) la experiencia propia, un sentido de lo que es lo colectivo, de lo que es lo solidario, de lo que es la puesta en común. Y esto es un aprendizaje para los chicos, absoluto, que luego se traslada a otros aspectos de vida, se traslada a la vida personal, en la familia, y se traslada a la escuela (integrante del Equipo de Coordinación, Mesa Redonda - 2015).

Como explicaba la exintegrante del Equipo Administrativo ya citada (Entrevista - 2014), por medio de este tipo de prácticas se incorporan contenidos fundamentales y a la vez difíciles de aprehender por otros medios:

Escuchar a otros. El saber que yo tengo un momento y el otro tiene otro momento, $y$ hay momentos en que lo hacemos juntos y hay momentos en que no, y hay momentos en que yo debo esperar y hay momentos en que el otro espera porque tengo que aparecer yo. Esto me parece como una cosa muy importante, y muy difícil de lograr de otra manera.

\section{Una perspectiva que busca romper estigmas}

Según explican sus integrantes, en esta necesidad de revertir el carácter expulsivo de la educación con el foco puesto en la enseñanza colectiva están implicados principios opuestos a las políticas que estigmatizan a las poblaciones en situación de vulnerabilidad. En palabras de uno de ellos,

esa mirada siempre la ponemos en oposición a otras miradas al respecto de algunas políticas públicas; algunas, por ejemplo, que se plantean en términos de prevención, entonces se supone que porque una población está en una situación socioeconómica determinada, de mayor grado vulnerabilidad, de pobreza, como se quiera llamar, entonces automáticamente se da esta operación de que se los considera peligrosos (Entrevista a integrante del Equipo de Coordinación - 2014).

En efecto, se cuestiona "una mirada que estigmatiza a la pobreza, que la pone como peligrosa, esa no nos interesa, y evidentemente sabemos que está presente". Tampoco se trata de "salvar almas"; el mismo interlocutor discute rotundamente esa perspectiva y explica que "hay como cuestiones más profundas que nos interesa romper, la lógica esta de la piedad, de la lástima, nos parece... Digamos, hay una apuesta fuerte". Finalmente, a la mirada piadosa o la mirada temerosa se suma otra que menosprecia:

tampoco nos interesa es esta mirada que decía antes, más de "bueno, es social", como si fuera en detrimento de la calidad, o una cosa medio piadosa. Que también en definitiva está medio instalada en la noción que estamos disputando, porque, por ejemplo, de algún modo nos han sugerido que esto, bueno, si es algo de mucha 
calidad como que no se puede apreciar, como que para ciertos sectores sociales hay que dar algo más masticado, algo más... Hay como una mirada muy... no sé cómo decirlo, que desprestigia, una connotación muy negativa. Y bueno, con eso también [se quiere] romper. Y después, bueno, hay como cuestiones más profundas que nos interesa romper, la lógica esta de la piedad, de la lástima, nos parece... Digamos, hay una apuesta fuerte (Entrevista a integrante del Equipo de Coordinación - 2014).

Frente a estas miradas, destaca el mismo interlocutor, "Simplemente sí consideramos que hay una experiencia estética interesante en la posibilidad de ser parte de una orquesta, y que ciertos sectores sociales, digamos, sabemos que han sido vedados".

En suma, este proyecto articula distintas perspectivas y prácticas que giran en torno a la idea de posibilitar el acceso a determinadas manifestaciones musicales a actores sociales que, por condiciones de desigualdad estructural, no suelen tenerlo. Estas perspectivas se centran también en modos específicos de relación y de enseñanza/aprendizaje que buscan revertir miradas estigmatizantes y relaciones de exclusión.

\section{El cuestionamiento de la hegemonía. Dos versiones}

Los proyectos y programas de orquestas infantiles y juveniles comenzaron a desarrollarse en una Argentina que Maristella Svampa (2010) ha caracterizado como sociedad excluyente; en este contexto - si bien con variaciones coyunturales - , a lo largo de las últimas décadas han apuntado a generar intervenciones en el campo de una problemática, la de la exclusión, que tiene múltiples dimensiones, entre ellas la social y cultural. He señalado que diversos autores identifican como parte de los procesos de exclusión la fragilidad e inestabilidad de lazos sociales - y exacerbación del individualismo - (Castel, 1997; Fittoussi y Rosanvallon, 1997; Autes, 2004). En este contexto, proyectos y programas de orquestas como los que he descripto aquí apuntan a reconstruir vínculos y a generar experiencias de inserción en colectivos.

De modo paralelo, como ya se señaló, la exclusión tiene también una dimensión cultural. A diferencia de ideas restringidas respecto de la cultura, que la conciben como un ámbito autónomo y separado del devenir social, un proyecto cultural debe reconocer que "lo que buena parte de la cultura hace es producir sujetos y producir (y reproducir) relaciones sociales” (Vich, 2014: 84); es esta idea $-\mathrm{y}$ la propuesta de pensar a la política cultural como agente de cambio social - lo que lleva a Víctor Vich a hablar de "desculturizar la cultura". Se trata entonces de un campo dinámico de disputas por los sentidos y las representaciones (Wright, 2010); en consecuencia, puede ser un espacio de reproducción de prácticas e imaginarios hegemónicos ${ }^{20} \mathrm{o}$, por el contrario, de construcción de contrahegemonía (Williams, 1980).

La descripción de las perspectivas que sustentan actores que diseñan y ponen en acción los proyectos y programas aquí presentados posibilita pensar en clave del desarrollo simbólico que se busca promover, los imaginarios que pretenden reforzar o torcer, los sentidos que pueden desenmascarar. Como dije, si bien estas perspectivas no son las únicas, se plasman en políticas públicas; esto significa que el Estado toma cursos de acción específicos para atender a demandas sociales y, al mismo tiempo, se forjan, refuerzan o minan representaciones sociales. Es en buena medida por ello que, ante cambios de lineamientos
20. La idea gramsciana de hegemonía supone un proceso por medio del cual los intereses de un grupo son apropiados por otros grupos; de ese modo, se consigue mediante el consenso aquello que en otras ocasiones se logra mediante la coerción directa (Gramsci, 1981; Brow, 1990). Se trata de prácticas y representaciones implicadas en la totalidad de la vida; como explica Williams (1980: 131), la hegemonía "Es un vívido sistema de significados y valores - fundamentales y constitutivos- que en la medida en que son experimentados como prácticas parecen confirmarse recíprocamente". 
21. Se trata de términos complejos y que constituyen objeto de disputas. Sin adentrarme en discusiones que exceden este artículo, es menester aclarar que: a) las tradiciones no son estáticas, son siempre dinámicas; b) tampoco son inmóviles ni están acotadas a espacios restringidos, pues las expresiones culturales circulan, son apropiadas de modos diferentes y van adquiriendo diversos significados de acuerdo con sus usos; c) los cambios, movimientos, apropiaciones, etc., ocurren en contextos generalmente desiguales en cuanto a (entre otras cosas) la posibilidad de imponer, elegir, discutir y cuestionar expresiones, valoraciones y usos. "Lo erudito", "lo culto", "lo popular", son construcciones sociales, divisiones en un universo de sentidos que con frecuencia valoriza y jerarquiza.

22. Cabe recordar que me estoy refiriendo aquí al plano del diseño; los efectos de estos diseños, sus modos de acción, sus dinámicas específicas y las experiencias que generan deben ser estudiados en territorio y serán cuestiones a describir y analizar en el futuro (actualmente nos encontramos realizando trabajo de campo en diversas orquestas). en materia política, económica y social, es usual en la cultura política local incorporar nuevos actores y/o apartar a los precedentes.

Específicamente en los casos desarrollados en los apartados anteriores podemos observar que se trata de un proyecto y un programa que, en el plano de su diseño - cómo se los piensa, cómo se los organiza - , poseen características en común y varias diferencias. Entre las primeras está el valor de la enseñanza colectiva, el carácter irrestricto del ingreso, el cuestionamiento a ciertos estigmas, la construcción de una política pública que apunta a sectores históricamente postergados, la perspectiva de que la experiencia musical - más si es colectiva - puede incidir de forma positiva en los participantes, entre otras. Todo ello puede ser pensado como acciones que buscan minar representaciones y prácticas excluyentes. En cuanto a sus diferencias, podemos señalar que uno de ellos tiene alcance nacional y el otro, local; además de tener implicancias numéricas, administrativas y organizativas, acrecienta la - existente en ambos casos - heterogeneidad poblacional. También difieren en cuanto a sus modalidades de gestión o, más exactamente, respecto de los agentes que las diseñan y gestionan. En un caso se trata de una gestión articulada entre dos o más agentes (pues depende del Ministerio de Cultura y otros actores, sean públicos o del tercer sector); y en el otro, de un modelo de gestión única (dependiente del Ministerio de Educación).

En especial me interesa revisar aquí las diferencias en cuanto al repertorio y a los instrumentos empleados. Pues el tratamiento que se hace de las prácticas musicales puede contribuir tanto a reforzar como a cuestionar jerarquías; tanto a estigmatizar como a (re)activar identificaciones. Aclarando que no hay una división tajante entre las llamadas música erudita y música popular, los instrumentos son, en un caso - y más allá de su origen - , los empleados usualmente en la música popular latinoamericana y, en el otro, los que integran las orquestas sinfónicas de tradición europea. ${ }^{21}$ Los enfoques pedagógicos según los cuales se parte de la oralidad en un caso, y de la notación musical en el otro, se vinculan con esas tradiciones (una escrita y la otra oral); asimismo, acompañan el repertorio, que en el primer caso es de expresiones musicales específicamente latinoamericanas y, en el segundo, abarca tanto la llamada música académica como la popular, desde el formato de la orquesta sinfónica.

Entiendo que, desde perspectivas parcialmente diferentes, desde un foco puesto en problemáticas diversas y desde diseños, en consecuencia, distintos, en ambos casos se está cuestionando un orden simbólico hegemónico que refuerza situaciones de desigualdad y exclusión. ${ }^{22}$ Se pueden señalar dos anclajes hegemónicos - entre los muchos existentes - que son puestos en entredicho: el primero de ellos tiene que ver con las expresiones que se valorizan desde el Estado. Si consideramos en clave histórica la construcción de identidades en el marco del Estado nación argentino y recordamos el imaginario construido por la generación de 1880 con base en la dicotomía "civilización o barbarie", podemos notar que, entre otras cosas, apuntó a construir una argentinidad europeizada que invisibilizaba a los "otros" internos. Como afirma Rita Segato, la "formación nacional de alteridad" de la Argentina se ha caracterizado por

la idea del "terror étnico", del patrullaje homogeneizador por parte de las instituciones y el trabajo estratégico de una elite portuaria e ideológicamente eurocéntrica en el control del Estado para “nacionalizar” una nación percibida como amenazadoramente múltiple en pueblos, y extranjera. Nacionalizar significó aquí moldearla en una especie de "etnicidad ficticia" férreamente uniformizada. El sujeto 
nacional tuvo que moldearse en un perfil neutro, vaciado de toda particularidad. “Civilización” fue aquí definida como "neutralidad étnica”, y "barbarie" como su antagónico otro interior en constante retirada y pugna por retorno (2007: 30-31).

En este contexto, prácticas musicales provenientes de - o asociadas con - esas alteridades que "debían" ser anuladas o escondidas han sido históricamente desvalorizadas, negadas o, en algunos casos, particularizadas. ${ }^{23}$ Entonces es allí donde cobra relevancia un programa que apunta a desplegar a nivel nacional prácticas musicales - las "especies", los instrumentos, las maneras de aprender y de hacer música - que, si bien tienen sus propios canales de expresión y difusión, han sido históricamente desvalorizadas y/o particularizadas. En oposición a esto, se las reivindica desde políticas culturales de nivel nacional para el conjunto de la población. Es decir, no solo se las revaloriza desde el Estado; también se reivindica el derecho de los diversos grupos sociales de la nación - y no solo los grupos que las generaron o con los que se las asocia - a apropiarse de estas expresiones. Expresiones que, como enfatizan desde el Programa Chazarreta, son "expresiones vivas".

El segundo anclaje hegemónico que se pone en entredicho es la clásica asociación, ya planteada por Bourdieu (2003), entre ciertas manifestaciones social e históricamente construidas como obras de $\operatorname{arte}^{24}$ y determinados grupos sociales que, por medio de la educación, adquieren una competencia artística que refuerza lo que él denomina "distinción". Se trata de la distinción entre quienes tienen el tiempo y los medios para tener el tipo de percepción estética sobre las obras de arte socialmente construida como válida y los que no los tienen; se legitiman y refuerzan así, dice Bourdieu, desigualdades económicas y sociales. Si bien coincido con autores que, como García Canclini (2010), observan que la popularización de museos y de expresiones de danza y música mediante diferentes medios de comunicación ponen en jaque este anclaje, no deja de ser cierto que ciertas expresiones artísticas de tradición occidental - la llamada "alta cultura" - contribuyen a "distinguir" a los grupos sociales que las conocen, consumen y ejecutan ${ }^{25}$ como poseedores de un capital cultural que los ubica "por encima" de otros. Ahora bien, ¿es una expresión - o una diversa serie de expresiones - mejor que otras? Por supuesto que no. Pero ¿por qué los distintos grupos que integran una sociedad no tienen las mismas posibilidades de acceso y apropiación?

Si retomamos de Bonfil Batalla (1984) la atención puesta en la posibilidad diferencial de los grupos sociales en cuanto al uso, producción y reproducción de elementos culturales, la ruptura del patrón según el cual solo ciertos grupos acceden a la producción de bienes simbólicos tales como la llamada música académica implica una restitución de derechos culturales. Reitero además que se trata de expresiones socialmente legitimadas. Sugiero entonces que se busca resquebrajar una articulación cristalizada según la cual solo las llamadas clases medias y altas pueden conocer, disfrutar y ejecutar ciertas manifestaciones de la tradición musical occidental. También se cuestiona otra distinción: aquella que muchas veces se enuncia entre música académica y popular - recordemos que, con instrumentos de la orquesta sinfónica, se ejecutan diferentes expresiones musicales - .

A partir de lo antedicho, considero que, si bien los diseños de estos programas en parte se contraponen, son necesarias ambas políticas para satisfacer necesidades culturales variadas, y para contribuir al desarrollo simbólico de una sociedad diversa y plural.
23. Explica Ana María Alonso (1994) que por medio de lo que ella denomina la "inscripción cultural” del Estado, se oculta el hecho de que este es una construcción histórica y se naturalizan los significados hegemónicos. Sus agentes e instituciones, entre otras cosas, buscan homogeneizar a la población incluyendo como nacionales ciertas prácticas y representaciones -y excluyendo otras-, al tiempo que particularizan ciertas prácticas como propias de grupos que no se consideran representativos de la Nación.

\section{Si bien Bourdieu atiende} especialmente a las artes plásticas, estas consideraciones se extienden a las diferentes manifestaciones estéticas históricamente asociadas con la "alta cultura", como las orquestas sinfónicas y la música académica.

25. Agradezco a la Dra. Paula Vilas la siguiente observación: no es un tema menor el hecho de que una buena parte de los recursos económicos provistos por el Estado para la música - sueldos de intérpretes en orquestas estables, de docentes en conservatorios, etc.- beneficie a músicos profesionales de formación académica. 
26. Dicen Bourdieu y Passeron (1996: 44) sobre la violencia simbólica: "Todo poder de violencia simbólica, o sea, todo poder que logra imponer significaciones e imponerlas como legítimas disimulando las relaciones de fuerza en que se funda su propia fuerza, añade su fuerza propia, es decir, propiamente simbólica, a esas relaciones de fuerza".

\section{Reflexiones finales}

He presentado y discutido aquí las perspectivas de integrantes de los equipos de coordinación de dos políticas públicas: el Programa Social Andrés Chazarreta (Ministerio de Cultura de la Nación) y el Proyecto Orquestas Infantiles y Juveniles (Ministerio de Educación de la Ciudad de Buenos Aires). En tanto son las posiciones - con diferencias, pero generalmente compartidas - de quienes se ocupan de los lineamientos de los proyectos o programas, son perspectivas que generan diseños y cursos de acción diversos y, en consecuencia, promueven imaginarios, relaciones y representaciones sociales. Hemos observado que en ambos casos se promueven modos de relación y acción que apuntan al fortalecimiento de vínculos, la inserción en colectivos, la posibilidad de participar en la producción y reproducción simbólica. En este sentido, se observa una intencionalidad de intervenir ante algunas de las dimensiones en juego en torno a la problemática de la exclusión.

Además de los lineamientos y miradas compartidas, se observan diferencias relativas a los objetivos, perspectivas, instrumentación y repertorio de los dos casos descriptos. El primero de ellos pone el foco en géneros musicales latinoamericanos, buscando hacer un contrapeso a la difusión hegemónica de la llamada música académica y otras expresiones de música popular que tienen sus propios canales de difusión, en particular de aquellas manifestaciones generadas o expandidas por la industria cultural. El segundo cuestiona la asociación histórica entre las elites y la música académica, sus instrumentos, su formación y sus saberes. Se quiebra la distinción que, según describe Bourdieu, refuerza relaciones de dominación entre grupos sociales.

Hemos observado a partir de allí que, desde ángulos que en parte se contraponen, ambas líneas de pensamiento y los focos que enfatizan generan el diseño de programas que buscan desestabilizar desde distintos ángulos órdenes simbólicos hegemónicos. Podríamos decir que se cuestionan dos tipos de violencia simbólica (Bourdieu y Passeron, 1996): ${ }^{26}$ por un lado, aquella que clasifica grupos y expresiones e indica que solo ciertos grupos pueden poseer ciertos conocimientos y practicar determinadas expresiones musicales; y por el otro lado, aquella que jerarquiza expresiones musicales y, junto con ellas, a las poblaciones con las que estas se asocian.

Es por ello que entiendo que si bien se trata de dos proyectos/programas que, por las concepciones que sustentan, implican diseños de políticas culturales diferentes y pese a que parecen propuestas opuestas, ambas buscan contribuir, desde sus diferentes paradigmas, a desestabilizar un orden simbólico que sustenta relaciones de desigualdad y exclusión.

\section{Agradecimientos}

Agradezco a los diferentes interlocutores por las charlas, la paciencia y la confianza, a las integrantes del grupo de Investigación sobre Música e Inclusión por los intercambios y el trabajo conjunto, y en especial a Javi por la lectura de este trabajo y por compartir miradas y experiencias. 
1 Existen investigaciones sobre este tipo de proyectos a nivel internacional; sobresalen por el alcance y la cantidad aquellas que observan el Sistema Nacional de Orquestas y Coros Juveniles e Infantiles de Venezuela — fundado por José Antonio Abreu en los años setenta- (Borchet, 2012; Majno, 2012; Tunstall, 2012; Uy, 2012; Baker, 2014). Pero también hay interesantes estudios sobre los proyectos de Chile (Velasco San Martín, 2009; Garat Ly, 2010), Escocia (Allan, 2010), Sudáfrica (Devroop, 2008; Roy, Devroop y Getz, 2015) o de diferentes continentes (Majno, 2012), por mencionar solo algunos. (En página 96.)

3 Es un equipo interdisciplinario de investigación radicado en la Universidad Nacional de Avellaneda, compuesto por investigadoras formadas y en formación de las áreas de Antropología, Ciencias Políticas, Gestión Cultural, Etnomusicología, Pedagogía Musical y Periodismo. Este equipo incluye, además de la autora de este artículo, a Eugenia Amantía, Gabriela Barro Gil, Alina Cibea, Gladys Giliberti, Camila Juárez, Elsa Martínez, Verónica Talellis y Paula Vilas. Agradezco especialmente las discusiones que he tenido con Verónica Talellis y Alina Cibea, que contribuyeron significativamente a las ideas que aquí se vuelcan. (En página 97.)

4 Desde ya, los diferentes integrantes de un mismo proyecto pueden también sustentar posiciones diversas -en general, esto ocurre dentro de ciertas ideas compartidas-. Esto se dificulta -o enriquece, según el caso- cuando, siguiendo cambios políticos coyunturales, se pone a la cabeza de un proyecto o programa alguien que no coincide con el resto de la gestión del proyecto; en esos casos puede haber diferencias entre los integrantes de los equipos tanto en el plano teórico como, en consecuencia, en las dinámicas de acción. No está de más aclarar entonces que de ninguna manera se considera a los programas como unidades monolíticas; en este artículo, sin embargo, me concentro en las miradas que dan forma a los diseños de los proyectos/programas. (En página 98.)

6 El corpus en el que se sustenta la investigación de la que es parte este trabajo excede las fuentes aquí empleadas e incluye, desde un enfoque etnográfico, entrevistas y comunicaciones informales que se vienen desarrollando desde el año 2014 con coordinadores, directores, docentes y estudiantes de diferentes programas, proyectos y orquestas, así como la observación participante en encuentros, clases, manifestaciones y conciertos. (En página 98.)

7 En esta mesa, referentes de diversos proyectos y programas de orquestas discutieron y pusieron en diálogo los procesos de creación, los desarrollos y las dinámicas cotidianas que implica gestionar este tipo de iniciativas. Si bien el grupo ha gestionado otros eventos vinculados con orquestas (conciertos, por ejemplo), no se realizó aún otra mesa redonda con esta dinámica, que tuvo como particularidad la búsqueda de un espacio donde cada referente de los distintos proyectos/programas pudiera presentar y poner en diálogo sus proyectos, perspectivas, modos de implementación, dificultades, etcétera. (En página 98.)

9 Si bien, como explican los mismos actores, un programa abarca un conjunto de proyectos bajo su órbita, mientras que un proyecto es más acotado, existen proyectos, como el que aquí estoy describiendo, que tienen características de un programa - muchas 
orquestas, la generación de diversas iniciativas que funcionan como proyectos dentro del proyecto, etc.- y viceversa (Avenburg, Cibea y Talellis, 2017). (En página 99.)

11 De acuerdo con integrantes del programa, el despido de Eduardo Tacconi a fines de 2017 pone en duda la continuidad de aquel. Esto ocurre en el contexto de diversos y conflictivos procesos de recortes presupuestarios y reducción de personal en diferentes áreas de Estado (también en el sector privado) como parte de los lineamientos políticoeconómicos de la gestión iniciada en 2015 por el presidente Mauricio Macri. En efecto, en forma posterior al envío de este artículo, el Programa Chazarreta dejó de funcionar como tal, y se diluyó en el marco del Nuevo Plan Nacional de Orquestas Infantiles y Juveniles. Este último fue presentado por el ministro de Cultura, Pablo Avelluto, en un concierto realizado en la Quinta Presidencial de Olivos en marzo de 2018 (https:// www.cultura.gob.ar/nuevo-plan-nacional-de-orquestas-infantiles-y-juveniles_5499/). En tanto no hay certidumbre sobre la dinámica y organización que tendrán las orquestas del programa en el futuro, me refiero a él según las perspectivas y líneas de acción que ha tenido hasta finales de 2017. (En página 100.)

12 En el rastreo realizado con el Grupo de Investigación sobre Música e Inclusión (Avenburg et al., 2015), reconstruimos los objetivos, la población destinataria y el repertorio de diferentes proyectos y programas. En los casos en que esta información no estaba disponible en sus páginas Web u otros medios, la obtuvimos a partir de las entrevistas realizadas con sus coordinadores, quienes previamente a nuestra publicación corroboraron la reconstrucción realizada. (En página 100.)

13 En Avenburg, Cibea y Talellis (2017) nos referimos a las distintas modalidades en las que se coordinan diferentes agentes en términos de gestión articulada, entre las que incluimos diversas variantes de combinaciones entre actores. En ellas se pueden identificar variaciones en torno a tres ejes: como primer eje se distingue el sector al cual pertenecen los agentes involucrados en la gestión (público, privado y tercer sector); como segundo y tercer ejes, dentro del sector público podemos distinguir, por un lado, el nivel administrativo al que pertenece cada institución (nacional, provincial, local), y por otro lado, el área de políticas públicas bajo la cual dichas instituciones funcionan (Cultura, Educación, Desarrollo Social, Salud, etc.). (En página 100.) 


\section{Q Referencias bibliográficas}

" ALLAN, Julie. 2010. "Arts and the inclusive imagination: Socially engaged arts practices and Sistema Scotland". Journal of Social Inclusion, 1 (2): 111-122.

" ALONSO, Ana María. 1994. "The Politics of Space, Time and Substance: State Formation, Nationalism, and Ethnicity". Annual Review of Anthropology, 23: 379-405.

» ANTELO, Estanislao y ZANELLI, Marcelo. 2004. Informe Final. Orquestas infanto/juvenil (Lugano). Ciudad Autónoma de Buenos Aires: Dirección de Investigación, Secretaría de Educación.

" AUTES, Michel. 2004. “Tres formas de desligadura”. En: S. Karsz (Coord.). La exclusión: bordeando sus fronteras. Definiciones y matices. España: Gedisa. pp. 15-53.

" AVENBURG, Karen, CIBEA, Alina y TALELLIS, Verónica. 2017. “Las orquestas infantiles y juveniles del Gran Buenos Aires. Estudio descriptivo del panorama de proyectos y programas vigentes entre 2014 y 2015”. Foro de educación musical, artes y pedagogía 2 (2): $13-57$.

» AVENBURG, Karen, CIBEA, Alina, TALELLIS, Verónica, BARRO GIL, Gabriela, GILIBERTI, Gladys y MARTíNEZ, Elsa. 2015. Publicación online de "Rastreo de los Proyectos de Orquestas Infantiles y Juveniles del Gran Buenos Aires (Argentina)". http://musicainclusion.silk.col. (Último acceso: 15-05-17)

" BAKER, Geoffrey. 2014. El Sistema: Orchestrating Venezuela's Youth. Nueva York: Oxford University Press.

» BARBIERI, Nicolás, PARTAL, Adriana y MERINO, Eva. 2011. "Nuevas políticas, nuevas miradas y metodologías de evaluación. ¿Cómo evaluar el retorno social de las políticas culturales?”. Papers Revista de Sociología, 96 (2): 477-500.

" BAYARDO, Rubens. 2010. "Políticas culturales y derechos culturales: entre la retórica y la realidad". Revista de Investigaciones Políticas y Sociológicas, 9 (2): 55-64. http://www. usc.es/revistas/index.php/rips/article/viewFile/1056/986

》BELFIORE, Eleonora. 2002. "Art as a means of alleviating social exclusion: Does it really work? A critique of instrumental cultural policies and social impact studies in the UK". International Journal of Cultural Policy, 8 (1): 91-106.

»BONFIL BATALLA, Guillermo. 1984. “Lo propio y lo ajeno. Una aproximación al problema del control cultural”. En: A. Colombres (Comp.) La cultura popular. México: Premiá. pp.79-86

» BORCHET, Gustavo. 2012. "El Sistema: a subjectivity of time discipline”. En: E. Araújo y E. Duque (Eds.) Os tempos sociais e o mundo contemporâneo. Um debate para as ciências sociais e humanas. Universidade do Minho: Centro de Estudos de Comunicação e Sociedade, Centro de Investigação em Ciências Sociais. pp. 59-79.

" BOURDIEU, Pierre. 2003. "Sociología de la percepción estética". En: Creencia artística y bienes simbólicos. Elementos para una sociología de la cultura. Córdoba y Buenos Aires: Aurelia Rivera. pp. 65-84.

» BOURDIEU, Pierre y PASSERON, Jean-Claude. 1996. La reproducción. Elementos para una teoría del sistema de enseñanza. México: Distribuciones Fontamara.

" BROW, James. 1990. "Notes on Community, Hegemony, and the Uses of the Past". Anthropological Quarterly, 63 (1): 1-6. Traducción de la cátedra de etnolingüística de la Facultad de Filosofía y Letras, Universidad de Buenos Aires. 
» CASTEL, Robert. 1997. La metamorfosis de la cuestión social. Paidós: Buenos Aires.

" DEVROOP, Karendra. 2008. "Changing Lives through Participation in Band: The South African Musical Outreach Project”. National Band Association Journal, 49 (2): 6o-61.

»DILLON, Verónica. 2008. “Arte e inclusión social. Construcción de identidades en ámbitos no formales”. Arte e Investigación, 2 (6): 131-135.

»DILLON, Verónica y DELUCA, Rafael. 2012. “Interdisciplina, identidad e inclusión social en ámbitos no formales”. Concinnitas, 02 (21): 73-85.

» FERNÁNDEZ CALVO, Diana. s/f. “La integración social de los niños y jóvenes pobres, a través de la música: Proyectos de orquestas juveniles en América Latina y en la Argentina”. http://www.especialmentemusica.com.ar/descargas/articulos_varios/articulo_varios_13.pdf

» FITOUSSI, Jean-Paul y ROSANVALLON, Pierre. 1997. La nueva era de las desigualdades. Buenos Aires: Manantial.

» GARAT LY, Giselle. 2010. “Las orquestas infantiles como herramienta para democratizar el acceso a una educación de calidad”. Ponencia presentada en el Congreso lberoamericano de Educación. 13 al 15 de septiembre. Buenos Aires.

» GARCÍA CANCLINI, Néstor (Ed.). 1987. “Políticas culturales y crisis de desarrollo: un balance latinoamericano”. En: Políticas culturales en América Latina. México: Grijalbo. pp. 13-53.

» GARCÍA CANCLINI, Néstor. 2010. La sociedad sin relato. Antropología y estética de la inminencia. Buenos Aires y Madrid: Katz.

» GRAMSCl, Antonio. 1981. Cuadernos desde la cárcel. Puebla: Universidad Autónoma de Puebla.

» GRECO, Lucrecia e IUSO, Gabriela. 2012. "En la roda, entre Brasil y Argentina. La capoeira en dos proyectos sociales latinoamericanos”. En: S. Citro y P. Aschieri (Coords). Cuerpos en Movimiento. Antropología de y desde las Danzas. Buenos Aires: Biblos/ Culturalia. pp. 219-233.

"INFANTINO, Julieta. 2011. “Artes entre políticas culturales e intervenciones sociopolíticas en Buenos Aires”. Nómadas, 34: 12-30. http://www.redalyc.org/articulo. oa? id=105118960002

» INFANTINO, Julieta y RAGGIO, Liliana. 2007. “La identidad de los jóvenes artistas circenses. ¿Cómo se construyó en diálogo con las políticas culturales estatales?”. Ponencia presentada en las VII Jornadas de Estudio de la Narrativa Folclórica. ISFNR Interim Conference. 20 al 22 de septiembre. Santa Rosa, La Pampa, Argentina. pp. 1-13.

» KARZS, Saül. 2004. “La exclusión: concepto falso, problema verdadero”. En S. Karzs (coord.): La exclusión: bordeando sus fronteras. Definiciones y matices. España: Gedisa. pp. 133-214.

» KLIKSBERG, Bernardo. 2014. Otra economía es posible. Desde el consenso de Washington a la visión de una nueva economía IV. Buenos Aires: La Página.

» LLORET, Vicenta. 2009. “Danza e integración”. Papeles de Arteterapia y educación artística para la inclusión social, 4: 79-96.

» MAJNO, Maria. 2012. "From the Model of El Sistema in Venezuela to Current Applications: Learning and Integration through Collective Music Education". Annals of The New York Academy of Science, 1252: 56-64. 
» MARTíNEZ, Elsa. 2016. “La música como forma de establecer vínculos interculturales. El caso de la Orquesta Infantil y Juvenil de Villa Inflamable (Avellaneda)". Ponencia presentada en el Primer Congreso Internacional de Artes. "Límites y fronteras en la escena artística contemporánea”. 20-22 de julio. Resistencia, Chaco, Argentina.

» MUIÑOS DE BRITOS, Stella Maris. 2010. “La práctica musical colectiva. Aprendizaje artístico y social”. Revista Iberoamericana de Educación, 52 (2). http://www.rieoei.org/ deloslectores/3589Muinos.pdf

» OLMOS, Héctor Ariel. 2008. Gestión cultural e identidad: claves del desarrollo. Madrid: Agencia Española de Cooperación Internacional para el Desarrollo.

" OSZLAK, Oscar. 2009. "Implementación participativa de políticas públicas: aportes a la construcción de un marco analítico”. En: A. Belmonte et al. (ed.) Construyendo confianza. Hacia un nuevo vínculo entre Estado y Sociedad Civil, Volumen II. Buenos Aires: CIPPEC y Subsecretaría para la Reforma Institucional y Fortalecimiento de la Democracia, Jefatura de Gabinete de Ministros, Presidencia de la Nación.pp. 9-47.

» OSZLAK, Oscar y O’ DONNELL, Guillermo. 1981. “Estado y Políticas Estatales en América Latina: Hacia una estrategia de investigación”. Centro de Estudios de Estado y Sociedad (CEDES). Buenos Aires, Documento G.E. CLACSO No 4.

»PÉREZ RUBIO, Ana María. 2006. “Acerca de la exclusión y otras cuestiones próximas”. Revista de Estudios Regionales, y Mercado de Trabajo, 2. http://www.simel.edu.ar/archivos/documentos/RS2\%2oRubio.pdf

» ROY, Michael, DEVROOP, Karendra y GETZ, Laura. 2015. “Improvement in South African students' outlook due to music involvement”. Music Education Research 17, Issue 4: 465-479. http://dx.doi.org/10.108o/14613808.2014.910183

» SÁNCHEZ, Romina. 2014. “Teatro comunitario y transformación social: La práctica artística en el proceso de construcción de una hegemonía alternativa”. Trabajo presentado en VIII Jornadas de Sociología de la UNLP, 3 al 5 de diciembre. Ensenada, Argentina. http://www.memoria.fahce.unlp.edu.ar/trab_eventos/ev.4219/ev.4219.pdf

»SEGATO, Rita. 2007. La nación y sus otros: raza, etnicidad y diversidad religiosa en tiempos de políticas de la identidad. Buenos Aires: Prometeo.

»SMALL, Christopher. 2010. Música. Sociedad. Educación. Madrid: Alianza.

»SVAMPA, Maristella. 2010. La sociedad excluyente. La Argentina bajo el signo del neoliberalismo. Buenos Aires: Taurus.

» TUNSTALL, Tricia. 2012. Changing Lives: Gustavo Dudamel, El Sistema, and the Transformative Power of Music. Nueva York: W. W. Norton \& Company.

» UY, Michael. 2012. “Venezuela's National Music Education Program El Sistema: Its Interactions with Society and its Participants' Engagement in Praxis”. Music and Arts in Action, 4 (1): 5-21.

» VÁZQUEZ, Alejandra. 2016. "Políticas públicas y perspectiva de género, análisis de un caso: El Programa Nacional de orquestas y coros para el bicentenario”. Ponencia presentada en las Jornadas de Investigación Antropología Social Santiago Wallace (FFyL, UBA). 27 al 29 de julio. Buenos Aires, Argentina.

»VELASCO SAN MARTíN, Patricia. 2009. Orquestas Juveniles e Infantiles de Chile: Una estrategia de Inclusión. Estudio de caso sobre las Orquestas de Curanilahue (Tesis de Magíster en Antropología y Desarrollo, Facultad de Ciencias Sociales, Universidad de Chile). http://www.tesis.uchile.cl/tesis/uchile/2009/cs-velasco_p/pdfAmont/csvelasco_p.pdf 
» VICH, Víctor. 2014. Desculturizar la Cultura. La gestión cultural como forma de acción política. Buenos Aires: Siglo XXI.

"VILLALBA, María. 2010. “La política pública de las orquestas infanto-juveniles”. Revista Latinoamericana de Ciencias Sociales, Niñez y Juventud 8 (1): 131-149. http://www.redalyc.org/pdf/773/77315079006.pdf

"VILLARREAL, Juan. 1996. La exclusión social. Buenos Aires: Norma.

»WALD, Gabriela. 2009. “Los dilemas de la inclusión a través del arte: tensiones y ambigüedades puestas en escena". Oficios Terrestres, 24: 53-63.

"WALD, Gabriela. 2011. "Promoción de la salud integral: el caso de dos programas de orquestas juveniles de la Ciudad de Buenos Aires". Revista Argentina de Salud Pública, 2 (7): 6-11.

"WALD, Gabriela. 2017. “Orquestas juveniles con fines de inclusión social. De identidades, subjetividades y transformación social”. Foro de Educación Musical, Artes y Pedagogía, 2 (2): 59-81.

»WILLIAMS, Raymond. 1980. Marxismo y Literatura. Barcelona: Península.

"WRIGHT, Susan. 2007. “La politización de la cultura”. En M. Boivin, A. Rosato y V. Arribas (Eds.). Constructores de otredad: una introducción a la antropología social y cultural. Buenos Aires: EUDEBA. pp. 128-141.

\section{Sitios Web/otras fuentes}

»Programa Social Andrés Chazarreta: https://chazarreta.cultura.gob.ar/

" Área de Orquestas y Ensambles Infantiles y Juveniles (en el que se inserta el Programa Social Andrés Chazarreta): https://altoque.cultura.gob.ar/

»Área Escuela Abierta (en la que se inserta el Proyecto Orquestas Infantiles y Juveniles) http://www.buenosaires.gob.ar/educacion/escuelaabierta/orquestas-coros-infantiles$y$-juveniles 